\title{
Validity of Ultrasonography in Diagnosis of Carpal Tunnel Syndrome
}

\author{
Authors \\ Dr Managalanandan. $S^{1 *}$, Dr Cinchu Santappan ${ }^{2,}$ Dr Mathew George ${ }^{3}$, \\ Dr Geena Benjamin ${ }^{4}$ \\ ${ }^{1}$ Associate Professor, ${ }^{2}$ Resident, ${ }^{3}$ Assistant Professor, ${ }^{4}$ Professor \\ Department of Radiodiagnosis, Pushpagiri Medical College, Thiruvalla, Kerala \\ *Corresponding Author \\ Dr Mangalanandan
}

Associate Professor, Department of Radiodiagnosis, Pushpagiri Medical College, Thiruvalla, Kerala, India

\begin{abstract}
Introduction: Carpal tunnel syndrome (CTS), a common peripheral entrapment neuropathys due to impairment of median nerve function. Diagnosis of CTS is based on clinical signs and symptoms and confirmed by electrodiagnostic studies. Ultrasound imaging is noninvasive, offers high temporal and spatial resolutions and can provide dynamic anatomical information regarding structure and kinetics.

Aims and Objectives: 1.To find the validity of using ultrasonography before and after provocative exercises for diagnosed CTS patients with nerve conduction studies. 2. To find out the cut off values of median nerve cross sectional area in our population in diagnosis of CTS and to compare it with NCS using a Receiver Operating Characteristic (ROC) curve.

Methodology: This is a study of diagnostic test tool evaluation. 29 patients diagnosed as CTS with nerve conduction studies, were taken for ultrasound before and after exercise and studied the cross sectional area of median nerve at wrist and tabulated.

Results: the cut off values of median nerve area in diagnosis of moderate and severe CTS is calculated. Predictive power of median nerve cross sectional area also is calculated.

Conclusion: As per the present study, the mean value of cross sectional area is 0.108 (pre exercise) and 0.125 ( post exercise) is diagnostic.
\end{abstract}

\section{Introduction}

Carpal tunnel syndrome (CTS), a common peripheral entrapment neuropathy, is recognized as one of the most important causes of workplace morbidity. It is due to impairment of median nerve function. ${ }^{(1)}$ The carpal tunnel is bounded by transverse carpal ligament (TCL) on the volar side, and eight carpal bones on the dorsal side. Nine flexor digital tendons and the median nerve pass through the carpal tunnel at the wrist level. ${ }^{(1,2)}$ Median nerve is subject to compression against TCL by tensed overlying flexor digital tendons within the carpal tunnel, during wrist and fingers movement. Main symptoms are an uncomfortable numbness, tingling, and pain in the median nerve distribution of the hands. ${ }^{(1)}$

Carpal tunnel syndrome is an entrapment neuropathy involving the median nerve at the level of the carpal tunnel. Compression of the median nerve at the carpal tunnel due to a healed 
radial fracture was first described by Paget $^{2}$ in 1854. Marie and Foix $1913^{3}$ were the first to describe the median nerve compression beneath the transverse carpal ligament.

The canal is formed by the transverse carpal ligament and carpal bones, and CTS arises from compression of the median nerve in this canal. Under pathological circumstances tissue pressure in the carpal tunnel reaches $30 \mathrm{~mm} \mathrm{Hg}$; that is four times the normal level (Szabo and Chidgey 1989).

Factors contributing to the development of CTS are numerous and often coexist in individuals: Congenital smallness of the tunnel or anatomical peculiarities (persistent median artery, abnormal course or insertion of finger flexor and palmaris muscles); susceptibility of the nerve to pressure (diabetic or other neuropathies, etc); systemic and endocrine disorders (pregnancylhypothyroidism, acromegaly, amyloidosis, etc $)^{3}$

\section{Diagnosis by Nerve Conduction Study:} Neurophysiological diagnosis of carpal tunnel syndrome was established by Simpson in 1956 by demonstrating focal slowing of median nerve conduction at the wrist. Both sensory and motor conduction are abnormal but sometimes only one is affected. For the diagnosis of carpal tunnel syndrome, median nerve is compared with ulnar or radial nerve of the same limb. The following criteria are suggestive of carpal tunnel syndrome ${ }^{4}$

\section{Diagnosis by uitrasonography}

Ultrasonographic diagnosis of carpal tunnel syndrome is based on multiple parameters. The main finding is the increase in cross sectional area of the nerve at site of compression. An area greater than $10 \mathrm{~mm}^{2}$ is suggestive of carpal tunnel syndrome. By new set of studies the predictive value of early evidence of progression of carpal tunnel syndrome is high. The increase in area of the median nerve (post exercise swelling ratio) after provocative exercise is said to have high sensitivity of $95 \%$.
As per literatures the post exercise swelling ratio has the highest sensitivity in diagnosing dynamic $\mathrm{CTS}^{5}$.

Value of sonography for the detection of median nerve entrapment inside the carpal tunnel was furthered in 1992 when the first systematic approach to the ultrasonographic diagnosis of CTS was reported (Buchberger et al. $1992^{6}$ ). Since then several reports in the literature have focused on the value of different ultrasonographic criteria for CTS diagnosis (Duncan et al. 1999; Lee et al. 1999; Nakamichi and Tachibana et al 2007; Sarria et al. 2000; Dilley et al. 2001; Jamadar et al. 2001, Wong et al 2002). Most of these studies focused mainly on three sonographic techniques:

1. Measurement of median nerve diameter or cross sectional area at distinct locations along the carpal tunnel $^{7,8}, 2$. Flattening ratio of median nerve and 3. Bowing of the flexor retinaculum.

Enlargement of the median nerve is a sign of CTS, there is some disagreement as to the amount of enlargement considered to be pathological. In the study by Buchberger et al. $\left(1991,1992^{6}\right)$ a cross sectional area of the median nerve of $10 \mathrm{~mm}^{2}$ measured in the proximal or middle carpal tunnel coincided with CTS Koyuncuoglu et al. (2005) ${ }^{9}$ reported a cross sectional area of $8.8 \mathrm{~mm} 2$ to be diagnostic in patients with positive clinical findings but negative electrodiagnostic testing.

A cut-off value of $>4 \mathrm{~mm}$ between the most anterior part of the carpal ligament and the base line between the hamate and trapezium has been reported to be significant for CTS (Buchberger et al. 1992) ${ }^{6}$

A distal flattening ratio above 3 has been defined as a cut-off value above which CTS has to be suspected (Buchberger et al. 1991, 1992 ${ }^{6}$ ).

Other parameters which have been studied for the diagnosis of CTS include a loss of fascicular discrimination in the enlarged median nerve (Lee et al. 1999; Nakamichi and Tachibana 2007; Mallouhi et al. 2006) together with marginal effacement from edema. Also an increase in internal and perineural vascularization as detected 
by increased flow on Doppler is suggestive of CTS $^{10}$

In 1988 B D Fornage ${ }^{11}$ used High-resolution realtime ultrasonography to evaluate peripheral nerves of the extremities in healthy subjects. The normal median and ulnar nerves in the upper extremity and the normal sciatic and external popliteal nerves in the lower extremity were seen, all having an echogenic fibrillar echotexture.

In 2003 Beekman et al14 reviewed the available literature on the topic and concluded that reliable diagnosis of CTS could be made ultrasonographically, mainly based on an increase in cross-sectional area of the median nerve at the level of the pisiform or hamate bone, based on seven studies

In 1988 B D Fornage11 used High-resolution realtime ultrasonography to evaluate peripheral nerves of the extremities in healthy subjects. The normal median and ulnar nerves in the upper extremity and the normal sciatic and external popliteal nerves in the lower extremity were seen, all having an echogenic fibrillar echotexture.

In 2004 Altinok et al $^{12}$ analysed the crosssectional areas (CSA), flattening ratios at three different levels, for the presence and the severity of CTS. Twenty had normal nerve conduction studies defined as mild, and 20 of them had abnormal NCS defined as moderate. The control group consisted of 20 healthy participants. All parameters were significantly different between patient and control groups. They concluded that detection of at least two of the three criteria (median nerve CSA $>9 \mathrm{~mm}^{2}$ at pisiform level, swelling ratio $\geq 1.3$, and palmar displacement $>2.5$ $\mathrm{mm}$ ) may be helpful for the verification of the diagnosis.

In 2007 Visser et $\mathrm{al}^{13}$ concluded that patients with a clinical diagnosis of CTS, the accuracy of sonography is similar to that for EMG and also that sonography is probably preferable because it is painless, easily accessible and preferred by the patients.

Cartwright S Michael et $\mathrm{al}^{2} 2012$ found that, neuromuscular ultrasound measurement of median nerve cross-sectional area at the wrist is accurate and may be offered as a diagnostic test for CTS Level A. Neuromuscular ultrasound probably adds value to electromagnetic studies when diagnosing CTS and should be considered in screening for structural abnormalities at the wrist in those with CTS

\section{Materials and Methods}

Study Type: Cross sectional study with diagnostic test tool evaluation

Period: 18 Months.

Sample Size: Assuming a sensitivity of $95 \%$ and specificity of $90 \%$ for ultrasound in comparison to a Gold standard of NCS, along with a positivity rate of $60 \%$, relative precision of $20 \%$ and alpha error of $5 \%$, sample size is 29 . Will be studied and correlated with the NCS.

Inclusion Criteria: All Patients who are clinically suspected of having CTS and referred for NCS and subsequently for ultrasound.

Exclusion Criteria: Patients with traumatic median nerve injury Patients who have previously undergone decompression surgery and patients with anomalies of the median nerve. Patients who are not willing for ultrasound evaluation of median nerve.

Data Source: 1) Data for the study will be collected by pre- prepared case proforma which will include the clinical history, clinical findings, ultrasound findings and NCS after getting informed consent from the patients willing for the study. 2) Ultrasound will be done with High resolution transducer (ML 6-15 Mhz) of the Premium ultrasound machine GE Logiq S 7 EXPERT from GE health care 3) NCS will be done on Machine NIHON KOHDEN

Statistical Analysis: Data entry will be done using Microsoft Excel and analyzed using SPSS 20.0. Sensitivity, Specificity, Positive predictive value, Negative predictive value,. Positive likelihood ratio for ultrasonography will be calculated before and after provocative exercises. ROC curve will be drawn using the data collected. 
$\mathrm{P}$ value of $<0.05$ will be taken as statistically significant.

Procedure: The wrist region of the patients assessed for median nerve cross sectional area 1) At the site of lesion as well as proximal and distal segments of the median nerve at the wrist. 2) The test is repeated after the following exercise 'cloth wringing' and 'bottle opening' for a period of 2 minutes in repetitive and continuous manner at an interval of 10 minutes.

\section{Observations and Results}

High resolution ultrasound of the wrist was done on patients who was having complaints and had undergone NCS and were referred for ultrasound to Department of Radiodiagnosis. A total of 29 cases were studied over the period of 18 months.

Distribution of carpal tunnel syndrome according to nerve conduction studies

Nerve conduction studies confirmed that 29 cases had carpal tunnel syndrome of various degrees. 5 cases were severe, 11 cases mild and 13 cases moderate.

Table 1: Percentage distribution of the sample according to NCV diagnosis

\begin{tabular}{|l|c|c|}
\hline NCV & Frequency & Percentage \\
\hline Mild & 11 & 37.93 \\
\hline Moderate & 13 & 44.82 \\
\hline Severe & 5 & 17.24 \\
\hline Total & 29 & 100 \\
\hline
\end{tabular}

Table 2: Percentage distribution of the sample according to age

\begin{tabular}{|l|c|c|}
\hline Age & Count & Percent \\
\hline$<40$ & 8 & 27.6 \\
\hline $41-50$ & 5 & 17.2 \\
\hline $51-60$ & 13 & 44.8 \\
\hline$>60$ & 3 & 10.3 \\
\hline Mean \pm SD & $49.9 \pm 12.9$ & \\
\hline
\end{tabular}

Table 3: Percentage distribution of the sample according to sex

\begin{tabular}{|l|c|c|}
\hline Sex & Count & Percent \\
\hline Male & 23 & 79.3 \\
\hline Female & 6 & 20.7 \\
\hline
\end{tabular}

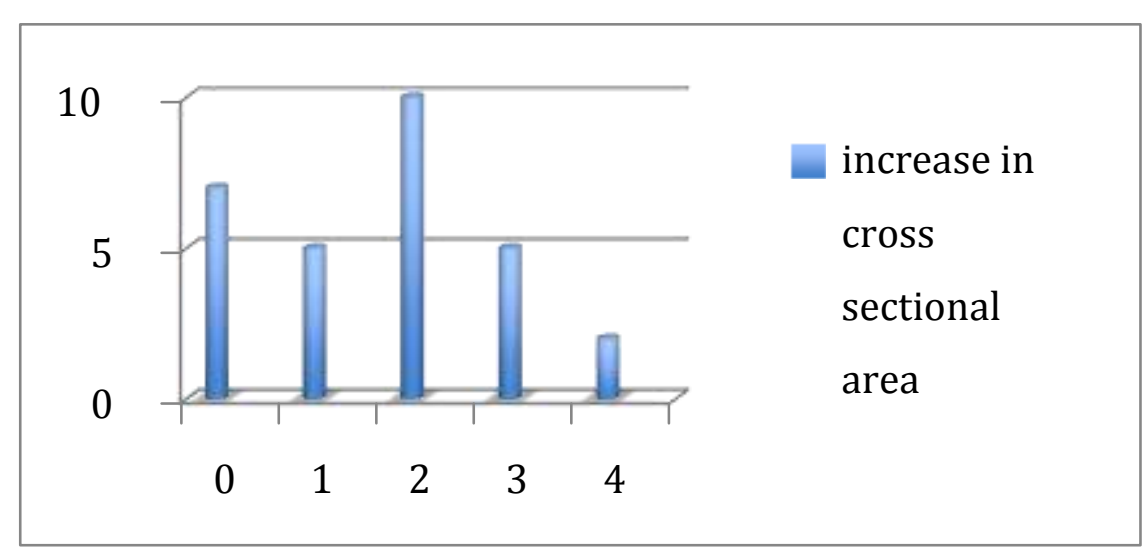

Fig 1: Increase in cross sectional area of the nerve after provocative exercise. 
As per the study 10 patients had a post exercise increase in nerve area of $2 \mathrm{~mm}^{2}$ and only 2 patients had nerve area increase of $4 \mathrm{~mm}^{2}$ and 7 patients had no significant increase in cross sectional area.

\section{Effectiveness of provocative exercises in nerve area}

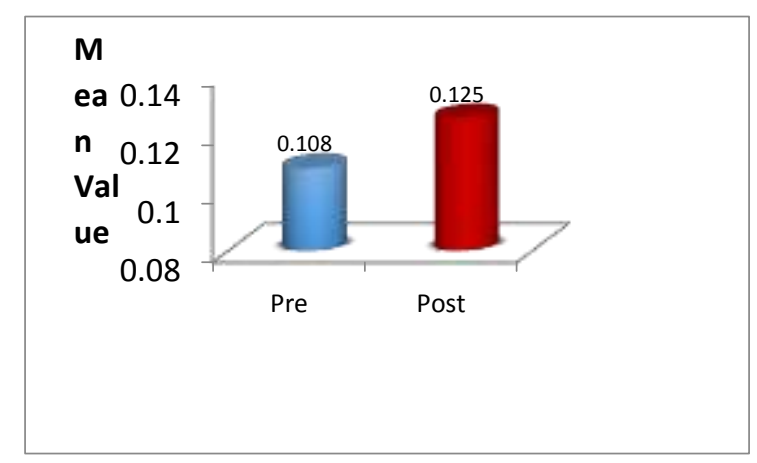

Fig.2 Effectiveness of provocative exercises in nerve area

The mean value of cross sectional area is 0.108 (pre exercise) and 0.125 (post exercise) as per the study.

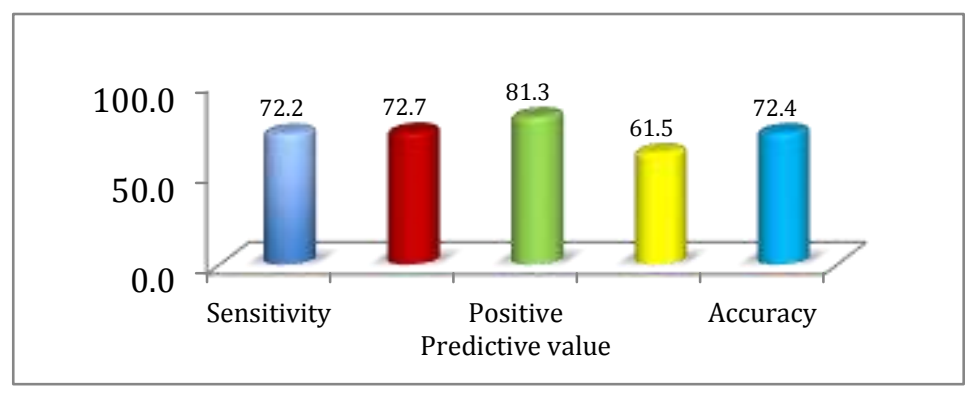

Fig. 3 Predictive power of median nerve area in diagnosis of Moderate / severe CTS

As per the study the predictive power of median nerve in diagnosis of moderate/ severe CTS, showed a sensitivity of 72.2 , specificity 72.2 , positive predictive value 81.3 , negative predictive value 61.5 and accuracy 72.4 .

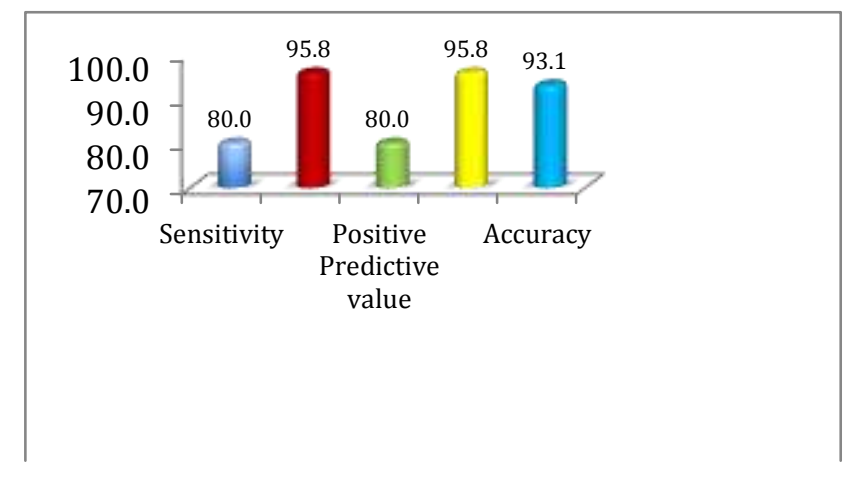

Fig. 4 Predictive power of median nerve area in diagnosis of severe CTS

As per the study, predictive power of median nerve in diagnosing of severe CTS showed a sensitivity of 80.0 , specificity of 95.8 , positive predictive value of 80.0 , negative predicitive value of 95.8 and accuracy of 93.1 . 
Background characteristics of patients

Effectiveness of provocative exercises in nerve area

Table 4: Effectiveness of provocative exercises in nerve area

\begin{tabular}{|l|c|c|c|c|c|c|}
\hline Nerve area & Mean & SD & N & Mean Difference & Paired t & $\mathrm{p}$ \\
\hline Pre & 0.108 & 0.04 & 29 & & & \\
\cline { 1 - 3 } & 0.125 & 0.04 & 29 & 0.016 & 6.46 & $\mathrm{p}<0.01$ \\
\hline
\end{tabular}

Find out the cut off values of median nerve area in diagnosis of CTS and to compare it with NCS using ROC curve

Find out the cut off values of median nerve area in diagnosis of Moderate / severe CTS Fig .5: ROC curve for prediction of CTS

Area Under the curve $=0.725,(0.54-0.91)(95 \% \mathrm{CI}), \mathrm{p}=0.045$, Best cut off $=0.11$

ROC Curve

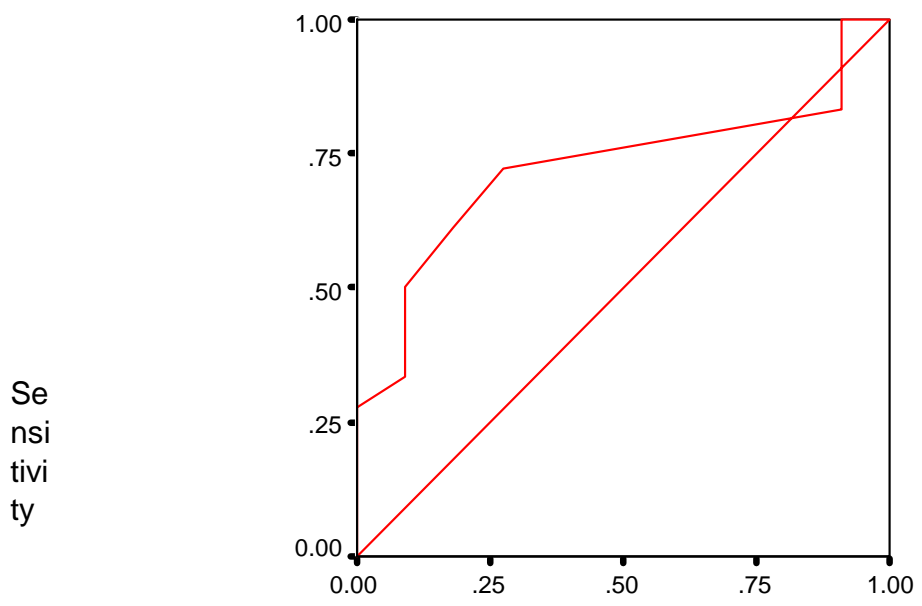

1 - Specificity Diagonal segments are produced by ties.

Table 5: Predictive power of median nerve area in diagnosis of Moderate / severe CTS

\begin{tabular}{|l|c|c|c|}
\hline \multirow{2}{*}{ Nerve area } & \multicolumn{3}{|c|}{ CTS } \\
\cline { 2 - 4 } & $\begin{array}{c}\text { Severe/ } \\
\text { Moderate }\end{array}$ & Mild & Total \\
\hline$>=0.11$ & 13 & 3 & 16 \\
\hline$<0.11$ & 5 & 8 & 13 \\
\hline Total & 18 & 11 & 29 \\
\hline
\end{tabular}

\begin{tabular}{|l|c|}
\hline Sensitivity & 72.2 \\
\hline Specificity & 72.7 \\
\hline False Negative & 27.8 \\
\hline False positive & 27.3 \\
\hline Positive Predictive value & 81.3 \\
\hline Negative Predictive value & 61.5 \\
\hline Positive Likelihood ratio & 2.6 \\
\hline Negative Likelihood ratio & 0.4 \\
\hline Accuracy & 72.4 \\
\hline
\end{tabular}


Find out the cut off values of median nerve area in diagnosis of severe CTS Fig.6 ROC curve for prediliction of CTS ROC curve for prediction of CTS

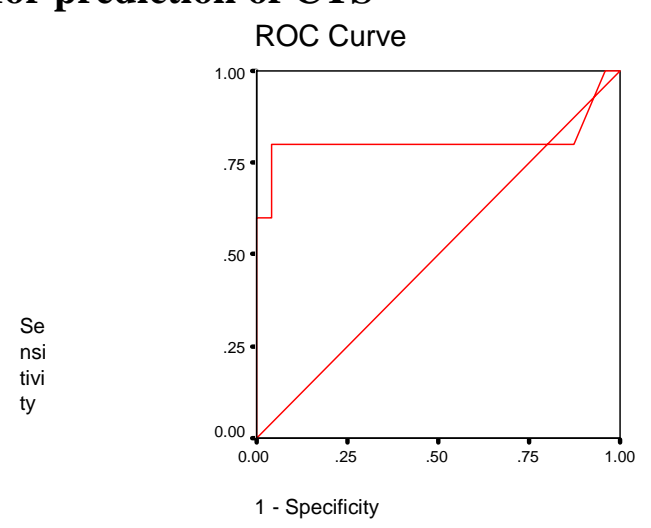

Diagonal segments are produced by ties.

Area Under the curve $=0.808,(0.49-1.13)(95 \% \mathrm{CI}), \mathrm{p}=0.033$, Best cut off $=0.17$

Table 6: Predictive power of median nerve area in diagnosis of severe CTS

\begin{tabular}{|l|c|c|c|}
\hline \multirow{2}{*}{ Nerve area } & & CTS & \\
\cline { 2 - 4 } & Severe & Mild/Moderate & Total \\
\hline$>=0.17$ & 4 & 1 & 5 \\
\hline$<0.17$ & 1 & 23 & 24 \\
\hline Total & 5 & 24 & 29 \\
\hline
\end{tabular}

\begin{tabular}{|l|c|}
\hline Sensitivity & 80.0 \\
\hline Specificity & 95.8 \\
\hline False Negative & 20.0 \\
\hline False positive & 4.2 \\
\hline Positive Predictive value & 80.0 \\
\hline Negative Predictive value & 95.8 \\
\hline Positive Likelihood ratio & 19.2 \\
\hline Negative Likelihood ratio & 0.2 \\
\hline Accuracy & 93.1 \\
\hline
\end{tabular}

As per the study conducted we have attained a sensitivity of $80.0 \%$ and specificity of $95.8 \%$ and accuracy of 93.1\%.

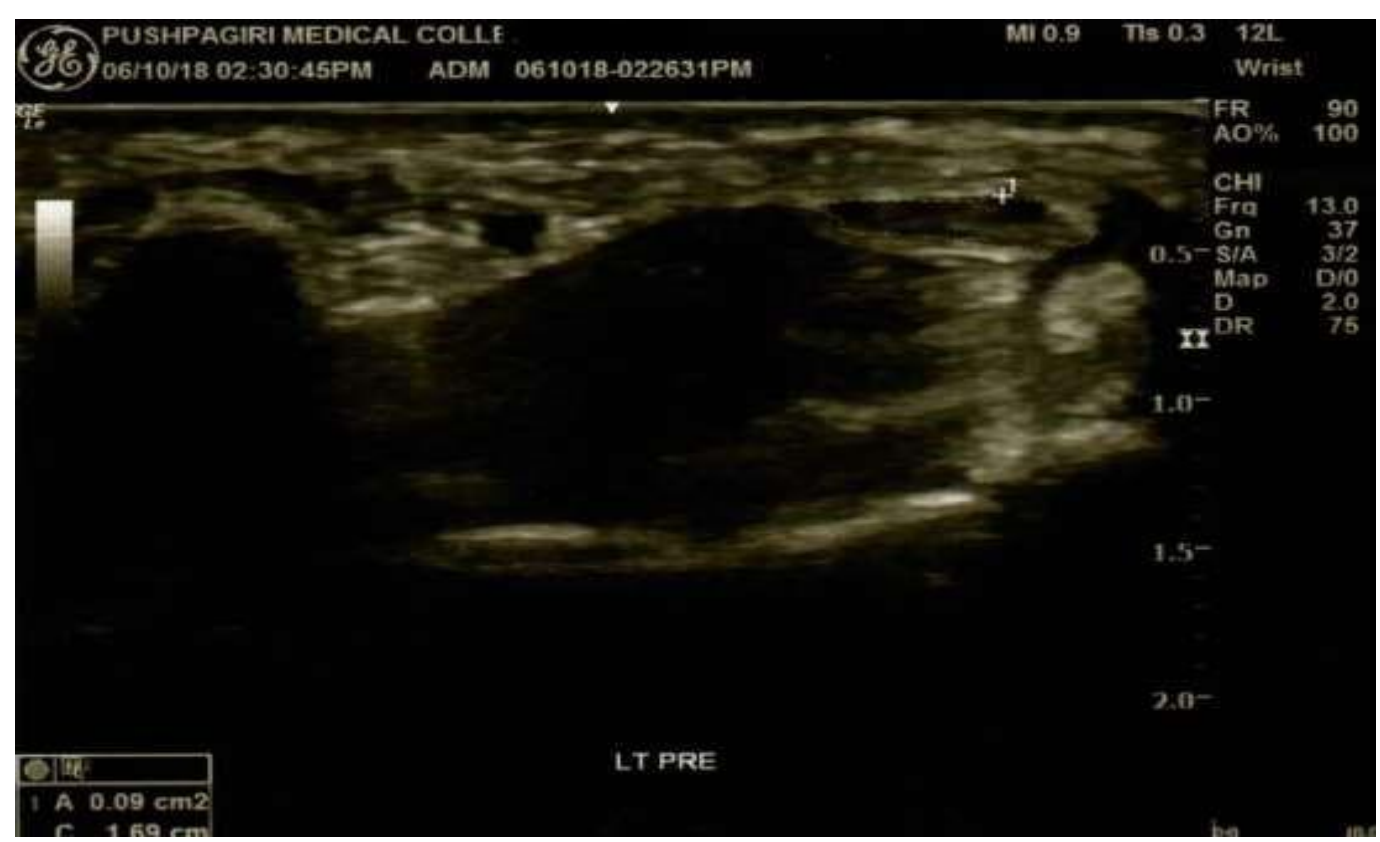

Figure 8: Size of median nerve before exercise in a 55 year old lady with CTS 


\section{JMSCR Vol||08||Issue||08||Page 319-329||August}

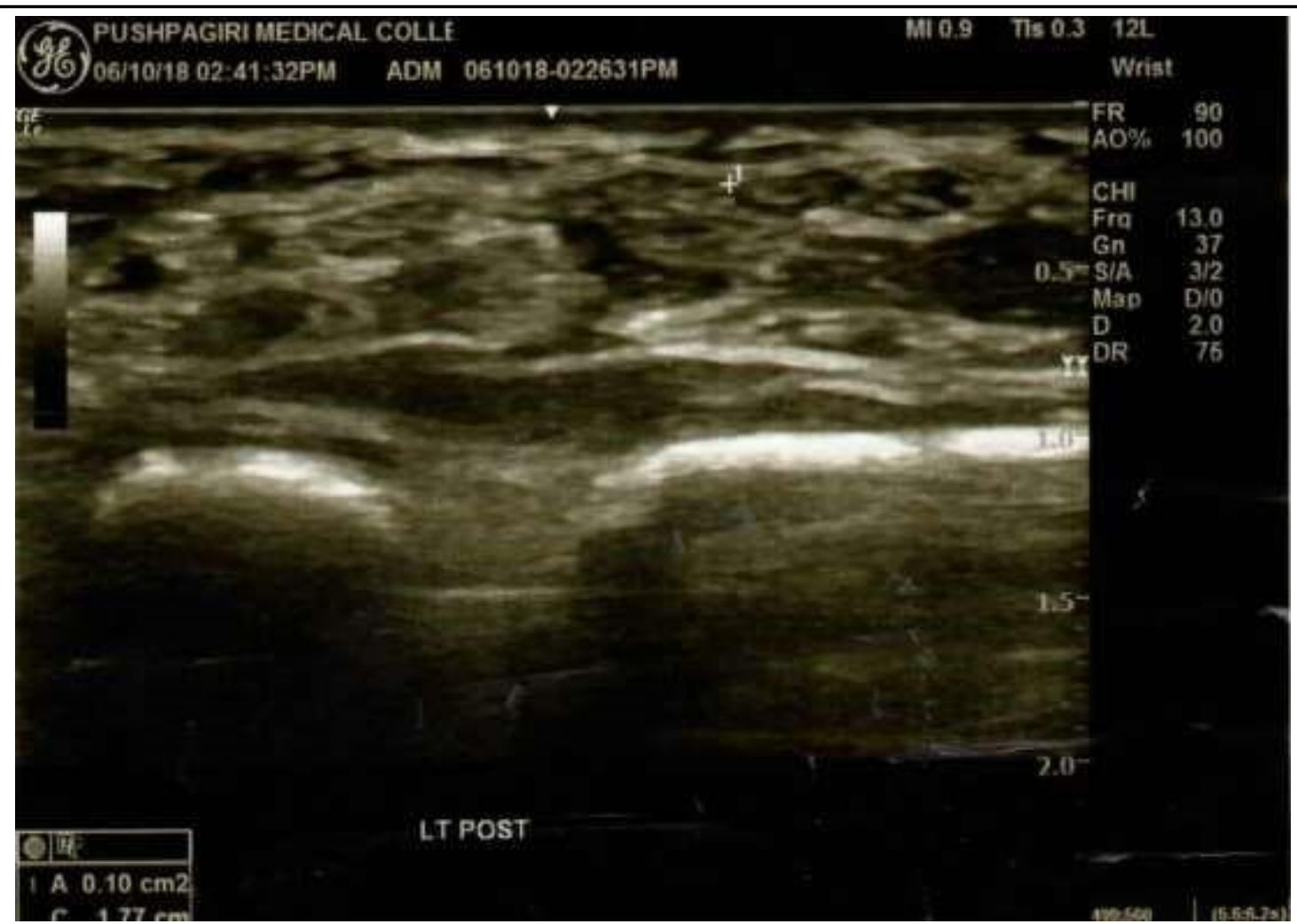

Figure 9: Increase in size of median nerve after exercise in a 55 year old lady with CTS

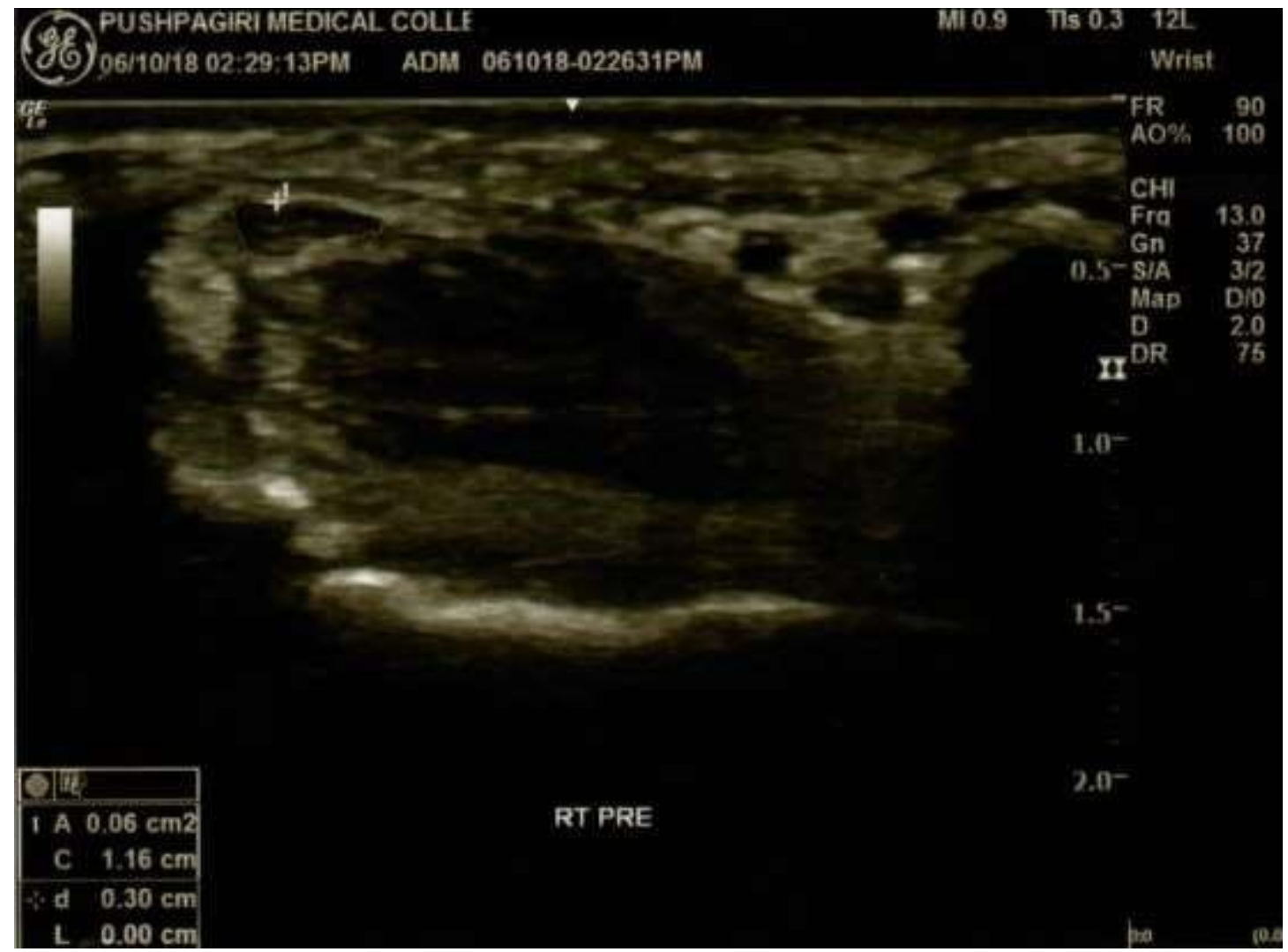

Figure 10: Size of median nerve before exercise in a 59 year old gentleman with CTS 


\section{JMSCR VoI||08||Issue||08||Page 319-329||August}

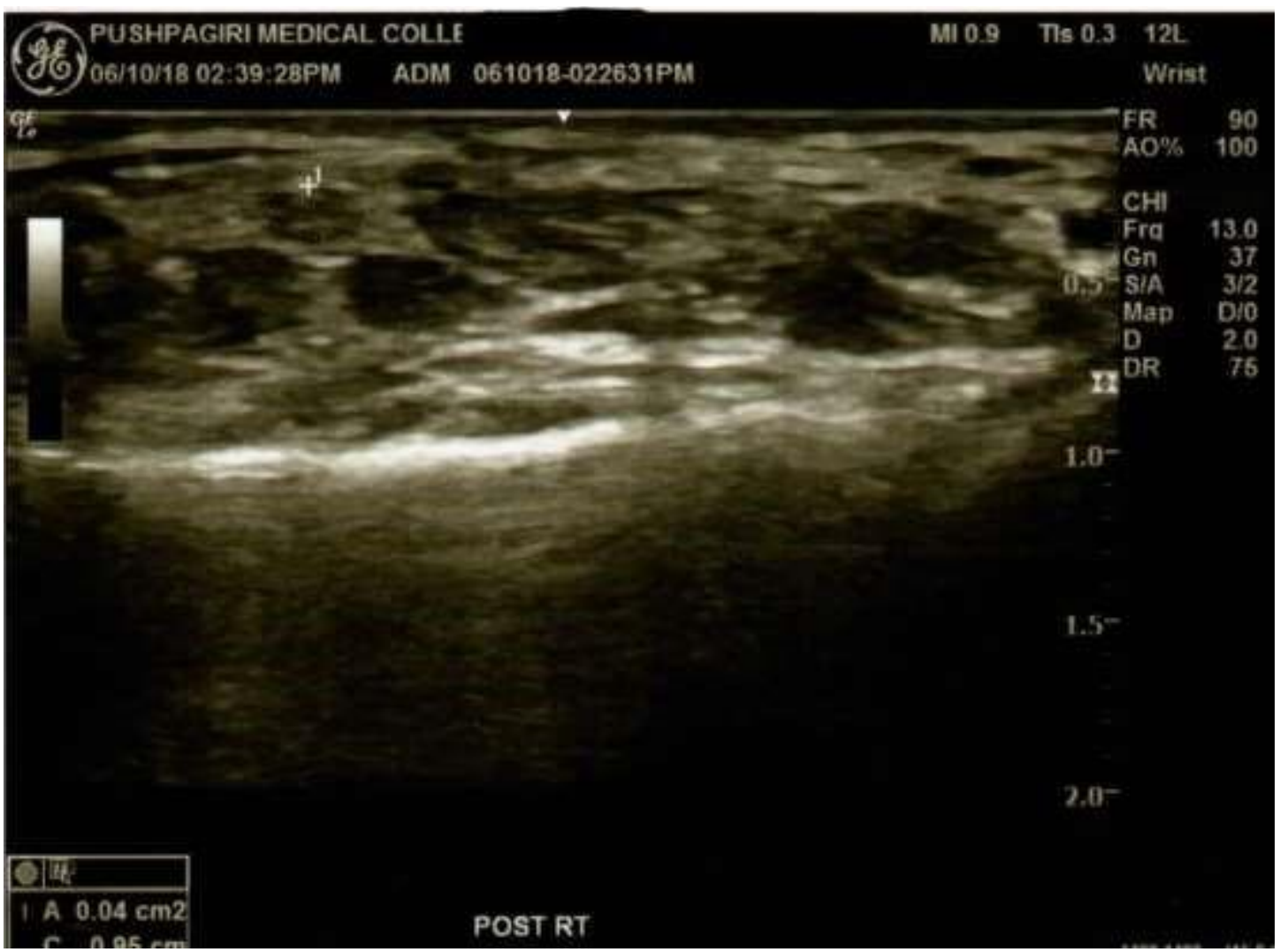

Figure 11: Increase in size of median nerve after exercise in a 59 year old gentleman with CTS

\section{Discussion}

Carpal tunnel syndrome patients evaluated in this study included cases clinically presenting with symptoms of wrist pain, in whom nerve conduction studies were done to serve as the gold standard diagnosis. In this study 29 cases were included which presented in the orthopaedics OP with wrist pain and had nerve conduction study done which is considered as the gold standard for carpal tunnel syndrome. Recent reports suggest that high resolution sonography can be used to diagnose carpal tunnel syndrome with the sensitivity and specificity approaching that of an electrodiagnostic study. The attraction of sonography for the diagnosis of CTS lies in its wide availability, low cost, non invasiveness, and shorter examination time.

Area of Median Nerve: Previous literature suggests that the area of the median nerve is one of the most sensitive and specific ultrasonological parameters that can be used for the diagnosis of carpal tunnel syndrome.

Altinok Tayfun $\mathrm{M}$ et $\mathrm{al}^{48}$ found that, in the preexercise period, there were no significant differences between patients and control subjects regarding palmar displacement and the swelling ratio. The difference was significant $(\mathrm{P}=.035)$ for the nerve area at the middle level. In the postexercise period, all parameters were significantly different between patients and control subjects. The nerve area at the middle level and the swelling ratio had higher significance $(\mathrm{P}<.0001)$ than palmar displacement ( $\mathrm{P}=.015)$. The postexercise swelling ratio had the highest sensitivity (95\%) in diagnosing dynamic CTS when a cutoff value of 1.26 was used. The provocative palmar displacement ratio had high sensitivity (80\%) and the highest specificity (90\%) for a cutoff value of 1.28 .

As per the study, the highest peak of CTS was noted between $51-60$ years $(44.8 \%)$ and second highest peak was noted between $<40$ years $(27.6$ $\%$ ) and least was noted in the age period of $>60$ years $(10.3 \%)$. So this suggest the prevelance of CTS in middle to ealderly age group.

The youngest patient was aged $29 \mathrm{yrs}$ and oldest was 79 years. 
Among the patients who were positive for CTS on NCS almost $79.31 \%$ were female, which is in comparison to previous literature which describes CTS as having a female preponderance.

As per the study 10 patients had a post exercise increase in nerve area of $2 \mathrm{~mm}^{2}$ and only 2 patients had nerve area increase of $4 \mathrm{~mm}^{2}$ and 7 patients had no significant increase in cross sectional area.

The mean value of cross sectional area is 0.108 (pre exercise) and 0.125 (post exercise) as per the study.

As per the study the predictive power of median nerve in diagnosis of moderate/ severe CTS, showed a sensitivity of 72.2 , specificity 72.2 , positive predictive value 81.3 , negative predictive value 61.5 and accuracy 72.4 .

Predictive power of median nerve in diagnosing of severe CTS showed a sensitivity of 80.0, specificity of 95.8 , positive predictive value of 80.0 , negative predicitive value of 95.8 and accuracy of 93.1 .

ROC curve was plotted and the predictive power of median nerve area in diagnosis of moderate/severe CTS calculated which showed a sensitivity of 72.2 , Specificity of 72.7 , false negativity 27.8 , false positivity 27.3 , positive predictive value 81.3 , negative predictive value 61.5, positive likelihood ratio 2.6,negative likelihood ratio 0.4 and accuracy of 72.4

A second ROC curve was plotted and the predictive power of median nerve area in diagnosis of severe CTS assessed, which showed a sensitivity of 80.0 , specificity of 95.8 , False negativity of 20.0, False positivity of 4.2 , Positive predictive value of 80.0, Negative predictive value of 95.8, Positive likelihood ratio 19.2, negative likelihood ratio 0.2 and an accuracy of 93.1.

\section{Limitations of the Study}

1. Relatively small sample size.

2. The study sample might not be true representative of the real world due to "sampling errors" and spectrum bias

\section{Conclusions}

As per the present study, the mean value of cross sectional area is 0.108 (pre exercise) and 0.125 (post exercise) is diagnostic. Predictive power of median nerve in diagnosis of moderate/ severe CTS, showed a sensitivity of 72.2, specificity 72.2. Predictive power of median nerve in diagnosing of severe CTS showed a sensitivity of 80.0, specificity of 95.8. Hence Ultrasonography cn be used effectively for the diagnosis of carpal tunnel syndrome.

\section{Bibliography}

1. Kuo Tzung - Tai, Lee RU -Ming, Liao Yin - Yin, Lee-Ning Wei, Hsu Wei Yen,Chen Perng - Jiann et al. Assessment of median nerve mobility by ultrasound dynamic imaging in carpal tunnel syndrome diagnosis. 2013 Joint UFFC, EFTF and PFM symposium.

2. Cartwright $\mathrm{S}$ Michael, Webb - Hobson D L, Boon J A, Alter E K, Hunt H C, Flores $\mathrm{H} \mathrm{V}$ et al - Evidence -Based Guideline: Neuromuscular Ultrasound for the diagnosis of Carpal tunnel Syndrome. American Association of neuromuscular and electrodiagnostic Medicine. Muscle Nerve 46: 287293,2012.

3. Rosenbaum RB, Ochoa JL (1993) Carpal tunnel syndrome and other disorders of the median nerve. Butterworth- Heinemann, Boston.

4. Mishra UK, Kalita J(1999)Clinical Neurophysiology, B.I. Churchill Livingstone Pvt limited,32-34

5. Altinok T, Baysal O, Karkas M H , Firat K A. - Sonographic Evaluation of the Carpal Tunnel after Provocative Exercises. J Ultrasound Med 2004;23:1301 - 1306.

6. Buchberger W, Judmaier W, Birbamer G et al. (1992) Carpal tunnel syndrome: diagnosis with high resolution sonography. AJR Am J Roentgenol 159:793-798

7. Nakamichi KI, Tachibana S. Enlarged median nerve in idiopathic carpal tunnel 
syndrome. Muscle Nerve 2000; 23:17131718.

8. Nakamichi K, Tachibana S. Ultrasonographic measurement of median nerve cross-sectional area in idiopathic carpal tunnel syndrome: diagnostic accuracy.Muscle Nerve 2002; 26:798-803

9. Koyuncuoglu HR, Kutluhan S, Yesildag A et al. (2005) The value of ultrasonographic measurement in carpal tunnel syndrome in patients with negative electrodiagnostic tests. Eur J Radiol 56:365-36

10. Mallouhi A, Pülzl P, Trieb T, Piza H, Bodner G (2006) Predictors of carpal tunnel syndrome: accuracy of gray-scale and color Doppler sonography. AJR Am J Roentgenol 186:1240-1245

11. Fornage BD. Peripheral nerves of the extremities: imaging with US. Radiology 1988; $167: 179-182$

12. Beekman R, Visser LH (2003) Sonography in the diagnosis of carpal tunnel syndrome: a critical review of the literature. Muscle Nerve 27:26-3

13. Fornage BD. Peripheral nerves of the extremities: imaging with US. Radiology 1988;167:179 -182

14. Altinok T, Baysal O, Karakas HM et al. (2004) Ultrasonographic assessment of mild and moderate idiopathic carpal tunnel syndrome. Clin Radiol 59:916-925

15. Visser LH, Smidt MH, Lee ML (2008) High-resolution sonography versus EMG in the diagnosis of carpal tunnel syndrome. J Neurol Neurosurg Psychiatry 79:63-67. 